\title{
Evaluación de la Satisfacción con el Tratamiento en un Centro Ambulatorio de Drogodependencias a través del "Treatment Perceptions Questionnaire" (TPQ)
}

\author{
Rodríguez, M. ${ }^{(1,7) ;}$ Jiménez-Lerma, J.M..$^{(2,7) ;}$ IRAURGI, I. ${ }^{(3)}$; MurUa, F. ${ }^{(4,7) ;}$ BacigaluPE, L. ${ }^{(5)}$; \\ ChavarRI, M.R. ${ }^{(6,7)}$; BalsategI, B. ${ }^{(6,7)}$ \\ (1) Psicóloga. Centro de Tratamiento de Toxicomanías de Alava. Vitoria-Gasteiz. Osakidetza/Servicio Vasco de Salud. \\ (2) Médico. Responsable del Centro Tratamiento Toxicomanías de Alava. Vitoria-Gasteiz. Osakidetza/Servicio Vasco de Salud. \\ (3) Psicólogo. Módulo de Asistencia Psicosocial de Rekalde. Bilbo-Bilbao. \\ (4) Directora de Enfermería del Hospital Psiquiátrico y de la Red de Salud Mental Extrahospitalaria de Alava. Osakidetza/Servicio Vasco de Salud. \\ (6) Directora de Personal del Hospital Psiquiátrico y de la Red de Salud Mental Extrahospitalaria de Alava. Osakidetza/Servicio Vasco de Salud. \\ (7) Enfermera. Centro de Tratamiento de Toxicomanías de Alava. Vitoria-Gasteiz. Osakidetza/Servicio Vasco de Salud. \\ (8) Asociación Vasca de Drogodependencias / Drogamenpekotasunerako Euskal Elkartea (AVD-DEE).
}

\section{RESUMEN}

Se valora mediante el 'Treatment Perceptions Questionnaire (TPQ)' el nivel de satisfacción reflejado por 100 usuarios de un Centro de Tratamiento de Toxicomanías, así como las posibles diferencias manifestadas en dos programas específicos del mismo: el Programa Libre de Drogas $(n=50)$ y el Programa de Mantenimiento con Metadona $(n=50)$. Para ello se realiza un estudio observacional de corte transversal donde los sujetos son seleccionados de forma sistematizada. Los resultados muestran un nivel aceptable de satisfacción, que se expresa en mayor medida entre los usuarios del programa libre de drogas tanto respecto a la puntuación total del instrumento como atendiendo a sus dos dimensiones: satisfacción con el programa y satisfacción con el equipo terapéutico. La comparación de los resultados de la muestra total con los datos ofrecidos en otras tres muestras europeas evidencia una equivalencia en las puntuaciones totales de satisfacción, pero diferencias en las puntuaciones por subdimensiones. EITPQ se muestra como un instrumento útil el cual nos permite con un mínimo de requerimiento de personal y de tiempo obtener una visión más clara de cómo nos perciben nuestros usuarios.

Palabras clave: Evaluación, Satisfacción, Usuario, Tratamiento, Drogas, cuestionario, TPQ.

\section{SUMMARY}

Satisfaction level reflected by 100 Drug Treatment Centre users is evaluated through a Treatment Perceptions Questionnaire (TPQ), in addition to the possible differences shown in two specific programmes within the TPQ: the Free Drugs Programme $(n=50)$ and the Methadone Maintenance Programme $(n=50)$. Cross section observational study is carried out with a systematic selection of subjects. The results show an acceptable level of satisfaction, expressed mainly among the drug-free programme users, with regard to the total instrument score, as well as its two dimensions: satisfaction with the programme and satisfaction with the therapist staff. Total sample results compared with three other European sample data show equivalence in the total satisfaction score, but differences in the sub-dimension scores. The TPQ is shown to be a useful instrument, which with a minimum number of personnel and time, enables us to obtain a clearer view of how our users see us.

Word Key: Evaluation, Satisfaction, User, Treatment, Drugs, questionnaire, TPQ.

\section{INTRODUCCIÓN}

$\mathbf{L}$ a calidad constituye una herramienta de gestión que tiende a una nueva forma de relación usuario-proveedor del servicio basada en la confianza del primero hacia el segundo, satisfaciendo, así mismo, tanto las necesidades y las expectativas de los usuarios como las del propio proveedor.(1-3)

Hoy en día existe un interés cada vez mayor por la calidad en el sector socio-sanitario que implica a todos los afectados: clientes, profesionales clínicos y gestores de servicios. La puesta en marcha de políticas y 
estrategias de calidad en el sector de las drogodependencias, que permitan ofrecer las suficientes garantías para la consecución de los objetivos asistenciales, suscita cada vez más interés.

Los estilos de gestión en las organizaciones han variado desde el Taylorismo de principios de siglo, basado en la "organización científica del trabajo", al sistema de gestión de calidad total (4) actual, el cual implementa la mejora continua en la organización y la implicación de todos sus miembros. Este nuevo modelo de gestión se centra en la satisfacción, tanto del cliente interno (profesionales de los recursos asistenciales) como del externo (usuarios, familiares, allegados), y dirige los recursos hacia las entidades que desarrollen tratamientos que tengan una eficacia demostrada

Las organizaciones de cualquier tipo o sector necesitan, para tener éxito, establecer un modelo de gestión apropiado. El modelo EFOM (European Foundation for Quality Management) de excelencia empresarial(5) es un instrumento práctico que ayuda a las organizaciones a establecer un sistema de gestión apropiado, midiendo en que punto se encuentra dentro del camino hacia la excelencia y analizando las diferencias y lagunas que pueden existir dentro de la organización para alcanzarla, estimulando la búsqueda de soluciones. Se orienta hacia el cliente, al que considera el árbitro final de la calidad del producto y del servicio, así como de decidir establecer una relación de fidelidad. El mejor modo de conseguir la fidelidad y retención del cliente es una clara orientación hacia las necesidades de los clientes actuales y potenciales.

El modelo EFQM consta de 9 criterios. Cinco agentes: a) liderazgo, b) política y estrategia, c) gestión de personal, d) alianzas y recursos, y e) procesos; y cuatro de resultados: f) satisfacción del cliente, g) satisfacción de las personas, h) impacto en la sociedad y i) resultados. El criterio 6 (f) mide los logros que está alcanzando la organización con relación a la satisfacción con sus clientes externos. Siendo su objetivo, examinar la forma en que el Centro conoce los niveles de satisfacción del cliente, e identifica y segmenta los diferentes grupos de clientes para mejorar la respuesta a sus demandas y expectativas razonables. Para ello es necesario manejar dos tipos de información, la relativa a la percepción del cliente, esto es, los términos subjetivos en que éste valora la calidad del servicio recibido, y la proporcionada por los indicadores objetivos de la gestión y funcionamiento de los servicios. La calidad de la atención que percibe el usuario está determinada básicamente por la diferencia entre las expectativas y la percepción de los resultados reales.

Son pocos los medios de participación que en este aspecto utilizan los servicios sanitarios. Para conocer aquello que quieren los usuarios se dispone, básicamente, de las reclamaciones y de las encuestas de satisfacción. A este respecto, la información sobre la percepción del usuario pueden obtenerse preguntándole mediante encuestas de valoración de servicios, las cuales comienzan a generalizarse en el sector socio-sanitario durante los últimos años. Por otra parte, la satisfacción que manifiesta el individuo respecto al tratamiento puede considerarse como un factor que modera el éxito del mismo, ya que es razonable esperar que los usuarios que no estén satisfechos pueden abandonar prematuramente o responder a las expectativas de modo diferente(6).

El objetivo del presente estudio es valorar el grado de satisfacción que tienen los usuarios de un centro ambulatorio de atención a las toxicomanías respecto al programa en el que se encuentran incluidos.

\section{MÉTODO}

Ubicación.- El estudio se ha desarrollado en el Centro de Tratamiento de Toxicomanías de Alava (CTT). Éste es un dispositivo sanitario público dedicado a la atención de usuarios diagnosticados de Trastornos por Abuso de Sustancias no alcohólicas en régimen ambulatorio. Durante el año 2001 fueron atendidos 666 usuarios distintos, 260 en programa de mantenimiento con agonistas opiáceos (PMM) y 406 en programa libre de drogas (PLD).

Población de estudio y muestra.- El estudio se centra en la valoración que han realizado 100 usuarios del centro, 50 incluidos en PMM y otros 50 en PLD, respecto a la satisfacción que tienen en cuanto a la atención y tratamiento recibido, medida con el 'Treatment Perceptions Questionnaire' (TPQ) (7).

Diseño y procedimiento.- El estudio se ha llevado a cabo mediante un diseño descriptivo de corte transversal utilizando métodos de autoinforme (cuestionario) para valorar la satisfacción con el programa.

El único criterio para participar en el estudio fue que los usuarios llevasen en el programa un mínimo de 15 días, para facilitar que los pacientes tuvieran un conocimiento mínimo respecto al funcionamiento de su programa de tratamiento. Dos monitores del centro se ocuparon de solicitar la participación, entregar el cuestionario y trasmitir las pautas de cumplimentación. Todos los individuos fueron informados de que se procedía a conocer cuál era su opinión respecto a la atención y características del centro y que se trataba de un cuestionario anónimo. La entrega de cuestionarios se realizó sistemáticamente hasta conseguir una muestra de 50 cuestionarios por programa; un total de 100 en el centro. Una vez contestado era introducido por el usuario en un buzón dispuesto a tal fin con objeto de respetar el anonimato. La participación en el estudio fue voluntaria y anónima. Seis usuarios se negaron a contestar el cuestionario. 
Instrumento.- El Treatment Perceptions Questionnaire (TPQ), desarrollado por Marsden, Gossop, Stewart et al (1998) (7), es un cuestionario destinado a población en tratamiento por adicción a drogas diseñado para medir la satisfacción del usuario respecto a su proceso terapéutico.

EITPQ es un cuestionario corto que tarda en administrarse aproximadamente 3 minutos. Está constituido por tres secciones, la primera "tu tratamiento" consta de 10 ítems con respuesta tipo likert de 5 puntos con un enunciado de respuesta que va desde 'muy de acuerdo' a 'muy en desacuerdo'. Cinco ítems están enunciados de forma positiva (p.ej.: He recibido la ayuda que buscaba) y los otros cinco de forma negativa (p.ej.: No me han gustado algunas de las reglas y normas del tratamiento), siendo recodificados los primeros de forma que una mayor puntuación indicaría un mayor nivel de satisfacción.

El análisis factorial del cuestionario que realizaron sus autores mostró dos dimensiones, cada una compuesta por cinco ítems. El primer factor recoge la percepción de los usuarios sobre la naturaleza y la intensidad del contacto con el personal del programa (Dimensión Equipo Terapéutico - ítems 1, 3, 4, 5 y 8) y el segundo factor agrupa los aspectos del servicio de tratamiento, su aplicación, reglas y normativas (Dimensión Programa Terapéutico - ítems 2, 6, 7, 9 y 10). Esta bidimensionalidad permite a los autores proponer la obtención de dos puntuaciones diferenciadas atendiendo al sumatorio de los cinco ítems que componen cada dimensión, oscilando las puntuaciones en cada caso entre 0 y 20 puntos. Asimismo, se puede obtener una puntuación total como resultado de la suma de las dos subescalas, o en su caso, por la suma total de los 10 ítems que componen el TPQ, situándose el recorrido de puntuaciones posibles entre 0 y 40 puntos.

Además, el TPQ incluye una segunda sección "sobre Ud." en la que recogen tres variables sociodemográficas (edad, sexo y tiempo de permanencia en tratamiento) siendo ampliadas en el presente estudio tanto las variables de tipo demográfico (sexo, edad, estado civil, nivel de estudio, situación laboral), como las que versan sobre antecedentes judiciales y aspectos relacionados con la historia de consumo de drogas (sustancia que genera la demanda de tratamiento, número de años de consumo, tiempo de permanencia en el programa terapéutico en meses y el año en el que iniciaron su primer tratamiento por abuso/dependencia de drogas). La tercera sección se denomina "este servicio" y recoge la opinión del usuario a través de una pregunta abierta. Esta última sección no ha sido considerada para análisis en el presente estudio.

Análisis.- Para la descripción de las características de la muestra y de los indicadores de satisfacción se utilizaron la frecuencia (n) y los porcentajes (\%) y/o los estadísticos de tendencia central (media -M- y desvia- ción estándar -DE-) según el tipo de variables consideradas, nominales vs intervalo, respectivamente. La hipótesis nula de no diferencia entre los programas terapéuticos respecto a las variables de caracterización se contrastó a través de la prueba de Ji cuadrado $\left(\chi^{2}\right)$, cuando las variables eran de tipo categorial, o mediante análisis de varianza ( $F$ de Snedecor) en el caso de variables continuas. Para el contraste de diferencias entre grupos respecto a las puntuaciones alcanzadas el cada uno de los ítems delTPQ, se utilizó la prueba $U$ de Mann-Whitney y su valor equivalente en puntuaciones Z, por tratarse de una medida de tipo ordinal. No obstante, el contraste de diferencias para las puntuaciones compuestas (sumatorio de la escala total y sus dos dimensiones) se realizó a través de análisis de varianza (prueba F o su equivalente $t$ de Student). Asimismo, las puntuaciones totales obtenidas con el conjunto de la muestra han sido comparadas con las observadas en tres estudios europeos(8), aplicando la prueba z que sigue una distribución normal y asumiendo un contraste bilateral. Todos los análisis se llevaron a cabo a través del programa estadístico SPSS(9) en su versión 9.

\section{RESULTADOS}

En las tablas 1 y 2 se recogen las variables de caracterización de la muestra total $(n=100)$, así como el contraste de diferencias respecto a las mismas entre los usuarios del PMM $(n=50)$ y del PLD $(n=50)$. El perfil general del usuario que ha participado en el estudio es de un hombre (77\%), con una edad media de 32,9 años ( $\mathrm{DE}=6,5$; recorrido de 18 a 48 años), soltero $(57 \%)$, que ha trabajado en mayor o menor medida durante el último año (54\%), y con un nivel educativo de bachiller elemental o graduado escolar (40\%). En el ámbito de problemas judiciales un 9\% no respondieron a esta cuestión, y de los que lo hicieron, el 62,6\% (57/91) no presentaban antecedentes penales. La heroína, consumida de forma única o en combinación con otra sustancia, fue la sustancia principal que generó la demanda de tratamiento (74\%); con una media de consumo de 11,6 años ( $D E=6,6$; recorrido 2 a 28 años). Los sujetos vienen recibiendo tratamiento en el CTT durante una media de 27,8 meses ( $D E=32$; recorrido 1 a 156 meses), e iniciaron su primer tratamiento de toxicomanías hace una media de 7,4 años ( $D E=6$; recorrido 0 a 26 años). En estas ultimas variables el índice de omisiones ha sido elevado.

Por grupos, se observan diferencias en la edad, la situación laboral, la presencia de problemas judiciales, la droga que genera la demanda, el tiempo de permanencia en el programa y el año en que iniciaron su primer tratamiento. A este respecto, los usuarios del PLD respecto a los usuarios del PMM, presentarían 


\begin{tabular}{|c|c|c|c|c|c|}
\hline \multicolumn{6}{|c|}{ Tabla 1. Variables de filiación. Características sociodemográficas CTT } \\
\hline & Total $(n=100)$ & PMM (n= 50) & PLD $(n=50)$ & Prueba de Contraste & p \\
\hline Edad-Media (Desv. Estándar) & $\begin{array}{c}32,95(6,49) \\
\mathrm{n} \approx \%\end{array}$ & $\begin{array}{c}34,62(5,31) \\
n(\%)\end{array}$ & $\begin{array}{c}31,28(7,15) \\
n(\%)\end{array}$ & $F_{(1,98)}=7,02$ & 0,009 \\
\hline \multicolumn{6}{|l|}{ Sexo } \\
\hline Hombre & 77 & $36(72 \%)$ & $41(82 \%)$ & \multirow[t]{2}{*}{$\chi^{2}=1,41$} & \multirow[t]{2}{*}{0,234} \\
\hline Mujer & 23 & $14(28 \%)$ & $9(18 \%)$ & & \\
\hline \multicolumn{6}{|l|}{ Estado Civil } \\
\hline Soltero/a & 57 & $28(56 \%)$ & $29(58 \%)$ & \multirow{5}{*}{$x^{2}=1,01$} & \multirow{5}{*}{0,909} \\
\hline Casado/a & 14 & $7(14 \%)$ & $7(14 \%)$ & & \\
\hline Convive en pareja & 16 & $7(14 \%)$ & $9(18 \%)$ & & \\
\hline Separado, Divorciado/a & 10 & $6(12 \%)$ & $4(8 \%)$ & & \\
\hline Viudo/a & 3 & $2(4 \%)$ & $1(2 \%)$ & & \\
\hline \multicolumn{6}{|l|}{ Estudios } \\
\hline Sin estudios & 4 & $1(2 \%)$ & $3(6 \%)$ & \multirow{6}{*}{$\chi^{2}=6,45$} & \multirow{6}{*}{0,264} \\
\hline Primarios-EGB & 20 & $11(22 \%)$ & $9(18 \%)$ & & \\
\hline Bach. elem.-Gr.Esc & 40 & $24(48 \%)$ & $16(32 \%)$ & & \\
\hline Bach. sup.-BUP-COU & 30 & $10(10 \%)$ & $20(40 \%)$ & & \\
\hline Universitarios & 3 & $2(4 \%)$ & $1(2 \%)$ & & \\
\hline No contestan & 3 & $2(4 \%)$ & $1(2 \%)$ & & \\
\hline \multicolumn{6}{|l|}{ Situación Laboral } \\
\hline 6 meses o más & 46 & $15(30 \%)$ & $31(62 \%)$ & \multirow[t]{9}{*}{$x^{2}=20,47$} & \multirow[t]{9}{*}{0,005} \\
\hline menos 6 meses & 8 & $6(12 \%)$ & $2(4 \%)$ & & \\
\hline paro & 11 & $5(10 \%)$ & $6(12 \%)$ & & \\
\hline Estudiante & 3 & $0(0 \%)$ & $3(6 \%)$ & & \\
\hline Pensionista & 13 & $11(22 \%)$ & $2(4 \%)$ & & \\
\hline Jubilado & 1 & $1(2 \%)$ & $0(0 \%)$ & & \\
\hline Labores del hogar & 2 & $2(4 \%)$ & $0(0 \%)$ & & \\
\hline Otros & 9 & $3(6 \%)$ & $6(12 \%)$ & & \\
\hline No contestan & 7 & $7(14 \%)$ & $0(0 \%)$ & & \\
\hline \multicolumn{6}{|l|}{ Problemas Judiciales } \\
\hline Sí & 34 & $12(24 \%)$ & $22(44 \%)$ & \multirow{3}{*}{$\chi^{2}=4,35$} & \multirow{3}{*}{0,036} \\
\hline No & 57 & $33(66 \%)$ & $24(48 \%)$ & & \\
\hline No Contestan & 9 & $5(10 \%)$ & $4(8 \%)$ & & \\
\hline
\end{tabular}

una menor edad, una mayor proporción de sujetos laboralmente activos, una mayor presencia de problemas judiciales, mayor variedad de sustancias como motivadoras de la demanda de tratamiento, una menor estancia en el programa terapéutico y un primer contacto con el tratamiento de su problema de drogas más próximo a la actualidad.

En la tabla 3 se presentan las puntuaciones obtenidas por la muestra total, y por cada uno de los grupos de tratamiento, tanto para cada uno de los ítems como para las tres puntuaciones compuestas derivadas del TPQ. Asimismo, la figura 1 muestra la distribución porcentual de las respuestas dadas para cada uno de los 10 ítems del cuestionario.

A nivel global, todas las puntuaciones medias obtenidas en el conjunto de ítems superan una puntuación de 2 , donde el recorrido posible está entre 0 y 4 . Ello quiere decir, que las puntuaciones medías tienden a ofrecer una valoración tendente a una mayor satisfacción. Las medias más bajas, entre 2,02 y 2,12, corresponden a los ítems con enunciado negativo. Atendiendo a la distribución de los porcentajes por niveles de respuesta (Figura 1), este efecto también se hace observable: los ítems formulados de forma positiva muestran un mayor porcentaje de las respuestas indicadoras 'de acuerdo', mientras que los enunciados en negativo tienden a equilibrarse entre ambos polos de respuesta 'de acuerdo' vs 'en desacuerdo'.

Se observan diferencias en la valoración que hacen los usuarios del PMM y del PLD en los ítems 1, 5, 8, 10 y en las tres puntuaciones compuestas. A este respecto, los sujetos del PLD mostrarían una mayor satisfacción tanto en la puntuación total como en la satisfacción mostrada al programa y al equipo que les 


\begin{tabular}{|c|c|c|c|c|c|}
\hline & Total $(n=100)$ & PMM $(n=50)$ & PLD $(n=50)$ & Prueba de Contraste & $\mathbf{p}$ \\
\hline \multicolumn{6}{|c|}{ Sustancia que genera la demanda } \\
\hline Heroína & 52 & $37(74 \%)$ & $15(30 \%)$ & $x^{2}=36,03$ & 0,000 \\
\hline Heroína + otra sustancia & 22 & $13(26 \%)$ & $9(18 \%)$ & & \\
\hline Cocaína & 8 & $0(0 \%)$ & $8(16 \%)$ & & \\
\hline Cocaína + otra sustancia & 10 & $0(0 \%)$ & $10(20 \%)$ & & \\
\hline Cannabis & 5 & $0(0 \%)$ & $5(10 \%)$ & & \\
\hline Cannabis y otras & 2 & $0(0 \%)$ & $2(4 \%)$ & & \\
\hline otras & 1 & $0(0 \%)$ & $1(2 \%)$ & & \\
\hline No contesta & 0 & $0(0 \%)$ & $0(0 \%)$ & & \\
\hline \multicolumn{6}{|l|}{ Años de consumo $(n=70)$} \\
\hline Media (Desviación Estándar & $11,6(6,6)$ & $13,4(6,4)$ & $10,3(6,4)$ & $F_{(1,68)}=3,86$ & 0,053 \\
\hline Rango & 2 a 28 & 2 a 26 & 2 a 28 & & \\
\hline No contestan & 30 & 23 & 7 & & \\
\hline \multicolumn{6}{|l|}{ Meses en el Programa $(n=80)$} \\
\hline Media (Desviación Estándar & $27,8(32,0)$ & $40,2(37,8)$ & $14,1(15,5)$ & $F_{(1,78)}=15,73$ & 0,000 \\
\hline Rango & 1 a 156 & 1 a 156 & 1 a 72 & & \\
\hline No contestan & 20 & 8 & 12 & & \\
\hline \multicolumn{6}{|l|}{ Año $1^{\circ}$ Tratamiento $(n=99)$} \\
\hline Media (Desviación Estándar & $1994,6(6,0)$ & $1991,4(6,0)$ & $1997,8(4,6)$ & $F_{(1,97)}=37,71$ & 0,000 \\
\hline Rango & 1976 a 2002 & 1976 a 2001 & 1983 a 2002 & & \\
\hline No contestan & 1 & 1 & 0 & & \\
\hline
\end{tabular}

Tabla 3. Puntuaciones en elTPQ. Diferencias por ítems y por subescalas entre el Programa de Mantenimiento con Metadona (PMM) y el Programa Libre de Drogas (PLD)

\begin{tabular}{|lccccc|}
\hline & Total (n= 100) & PMM (n= 50) & PLD (n= 50) & Prueba de Contraste & $\mathbf{p}$ \\
\hline Item 1 & $2,02(1,17)$ & $2,02(1,11)$ & $2,26(1,19)$ & $U=960,0 ; z=2,09$ & $\mathbf{0 , 0 3 6}$ \\
Item 2 & $3,23(0,68)$ & $3,18(0,71)$ & $3,28(0,64)$ & $U=1170,5 ; z=0,62$ & 0,531 \\
Item 3 & $2,03(1,17)$ & $1,86(1,14)$ & $2,20(1,18)$ & $U=1051,0 ; z=1,44$ & 0,149 \\
Item 4 & $2,94(1,14)$ & $2,88(1,20)$ & $3,00(1,08)$ & $U=1209,5 ; z=0,30$ & 0,764 \\
Item 5 & $2,63(1,11)$ & $2,36(1,12)$ & $2,90(1,03)$ & $U=888,0 ; z=2,64$ & $\mathbf{0 , 0 0 8}$ \\
Item 6 & $2,08(1,19)$ & $1,92(1,12)$ & $2,24(1,25)$ & $U=1066,0 ; z=1,32$ & 0,186 \\
Item 7 & $2,04(1,17)$ & $2,02(1,20)$ & $2,06(1,15)$ & $U=1226,0 ; z=0,17$ & 0,863 \\
Item 8 & $3,01(0,81)$ & $2,86(0,85)$ & $3,16(0,74)$ & $U=991,5 ; z=2,00$ & $\mathbf{0 , 0 4 4}$ \\
Item 9 & $2,96(0,81)$ & $2,84(0,76)$ & $3,08(0,75)$ & $U=1069,0 ; z=1,38$ & 0,167 \\
Item 10 & $2,12(1,21)$ & $1,82(1,24)$ & $2,42(1,11)$ & $U=908,0 ; z=2,46$ & $\mathbf{0 , 0 1 4}$ \\
& & & & \\
D. Equipo & $12,43(3,42)$ & $11,74(3,46)$ & $13,52(3,37)$ & $F_{(1,98)}=6,76 ; t=2,60$ & $\mathbf{0 , 0 1 1}$ \\
D. Programa & $12,63(3,14)$ & $11,78(3,13)$ & $13,08(3,14)$ & $F_{(1,98)}=4,29 ; t=2,07$ & $\mathbf{0 , 0 4 1}$ \\
Total & $25,06(5,87)$ & $23,52(5,89)$ & $26,60(5,86)$ & $F_{(1,98)}=6,86 ; t=2,62$ & $\mathbf{0 , 0 1 0}$ \\
\hline
\end{tabular}

PMM - Programa de Mantenimiento con Metadona; PLD - Programa Libre de Drogas

PRueba de Contraste: U -U de Mann-Whitney; F - F de Snedecor (análisis de varianza); $\mathrm{t}$ - $\mathrm{t}$ de Student; $\mathrm{p}$ - valor de probabilidad

ITEMS: 1) El equipo no siempre ha comprendido la clase de ayuda que yo quiero; 2) He sido bien informado acerca de las decisiones tomadas sobre mi tratamiento; 3) El equipo y yo hemos tenido ideas diferentes acerca de cuáles deberían ser los objetivos de mi tratamiento; 4) Siempre ha habido un miembro del equipo disponible cuando yo he querido hablar; 5) El equipo me ha ayuda a motivarme para solucionar mis problemas; 6) No me han gustado todas las sesiones de tratamiento a las que he asistido; 7) No he tenido tiempo suficiente para solucionar mis problemas; 8) Yo pienso que los miembros del equipo han sido buenos en sus tareas; 9) He recibido la ayuda que buscaba; 10) No me han gustado algunas de las reglas y normas del tratamiento. 
Figura 1. Distribución de los porcentajes por niveles de respuesta para los 10 ítems delTPQ

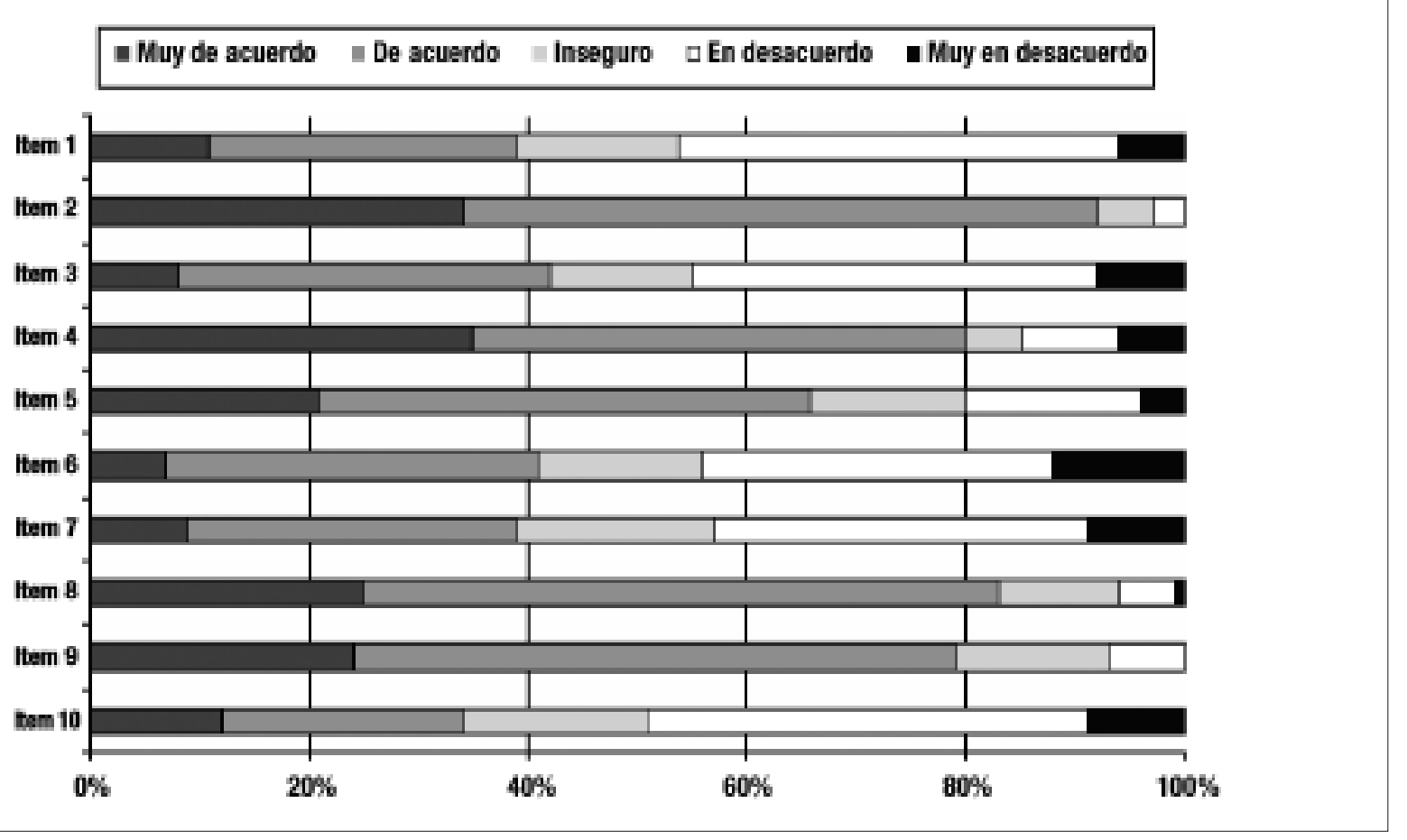

trata. Asimismo, y atendiendo a los ítems significativos, mostrarían una mayor percepción de que el equipo ha comprendido la clase de ayuda que precisaban (ítem 1), de que el equipo le ha ayudado a motivarse para solucionar sus problemas (ítem 5), de que los miembros del equipo son buenos en sus quehaceres (ítem 8) y han estado de acuerdo con las normas y reglas del programa (ítem 10). Nótese que los ítems 1 y 10 , con enunciado original en negativo, son valorados en sentido contrario al enunciado del ítem y ello es debido a la recodificación necesaria para el cálculo de las puntuaciones.

Finalmente, en la Tabla 4 se recogen las puntuaciones compuestas obtenidas en la muestra en estudio así como las ofrecidas en tres muestras europeas: una española, otra portuguesa y una última italiana. Asimismo, la Tabla 4 presenta el contraste de diferencias entre la muestra en estudio respecto a cada una de las otras tres. Tanto en el contraste con la muestra española como con el contraste con la muestra portuguesa se observan diferencias estadísticamente significativas en las dimensiones de satisfacción con el equipo y satisfacción con el programa, no así respecto a las puntuaciones totales. A este respecto, la muestra en estudio mostraría una mayor satisfacción en ambas dimensiones que la ofrecida por la otra muestra española, pero inferior a la satisfacción expresada por los portugueses. Por otra parte, respecto a la muestra italiana, las puntuaciones no presentan diferencias estadísticamente significativas.

Tabla 4. Comparación con estudios Europeos del TPQ.

\begin{tabular}{|c|c|c|c|c|c|c|c|}
\hline \multicolumn{8}{|c|}{ Comparación con estudios europeos } \\
\hline $\begin{array}{l}\text { Puntuaciones } \\
\text { delTPQ }\end{array}$ & $\begin{array}{l}\text { Vitoria } \\
\text { n (=100) }\end{array}$ & $\begin{array}{l}\text { España } \\
(n=45)\end{array}$ & $\begin{array}{c}\text { Contraste V-E } \\
z(p)\end{array}$ & $\begin{array}{l}\text { Portugal } \\
(n=27)\end{array}$ & $\begin{array}{c}\text { Contraste V-P } \\
\text { z (p) }\end{array}$ & $\begin{array}{l}\text { Italia } \\
(n=23)\end{array}$ & $\begin{array}{c}\text { Contraste V-I } \\
z \text { (p) }\end{array}$ \\
\hline D. Equipo & $12,6(3,1)$ & $11,3(2,7)$ & $4,87(<0,001)$ & $14,9(2,7)$ & $3,97(<0,001)$ & $13,5(3,0)$ & $1,28(0,202)$ \\
\hline D. Programa & $12,4(3,4)$ & $11,8(3,3)$ & $3,44(<0,001)$ & $14,4(3,3)$ & $2,69(0,007)$ & $11,8(4,1)$ & $0,56(0,574)$ \\
\hline Total & $25,1(5,9)$ & $23,1(5,3)$ & $1,48(0,138)$ & $23,3(5,3)$ & $1,10(0,272)$ & $25,3(6,2)$ & $0,09(0,928)$ \\
\hline
\end{tabular}




\section{DISCUSIÓN}

En general, los resultados obtenidos en nuestro estudio indican un nivel de satisfacción adecuado, al menos si los ponemos en relación con los resultados obtenidos en otras muestras europeas(8). Los cinco aspectos mejor valorados han sido a) la información recibida sobre las decisiones sobre su tratamiento, b) la eficacia del equipo terapéutico en sus tareas, c) haber recibido la ayuda que se buscaba, d) la disponibilidad del equipo para poder hablar con el paciente, y e) la ayuda recibida respecto a la motivación para la búsqueda de soluciones. Asimismo, se ha constatado diferencias en los niveles de satisfacción mostrados por los usuarios del PLD y/o del PMM, siendo los primeros los que manifiestan un mayor nivel de agrado tanto en la valoración total, como en las áreas relativas a la satisfacción con el programa y con el equipo terapéutico. No obstante, ha de constatarse que si bien estas diferencias han resultado ser estadísticamente significativas, la magnitud de las diferencias encontradas no alcanzar gran relevancia práctica; la máxima diferencia se alcanza en la puntuación total y es de 3,8 puntos sobre un rango posible de diferencias que oscila entre 0 y 40

Esta misma situación se produce en la comparación de los niveles de satisfacción mostrados en nuestra muestra respecto a los ofrecidos en otros estudios europeos. Las diferencias, en las áreas en las que muestran significación estadística, no superan los 3 puntos. En concreto, si consideramos la puntuación total del TPQ observamos que las cuatro muestras (española, portuguesa, italiana y la explorada en este estudio), ofrecen puntuaciones equivalentes. Las diferencias se hallan en las subdimensiones satisfacción con el programa y satisfacción con el equipo, donde los sujetos analizados en este estudio presenten una mayor satisfacción que otros usuarios españoles, pero menor que la expresada por usuarios portugueses. A este respecto, queremos llamar la atención sobre los datos ofrecidos para la muestra portuguesa por Marsden y colaboradores(8), ya que la suma de las subdimensiones no corresponde con la puntuación total, de lo cual inducimos que puede haber un error tipográfico en la publicación que debiera ser subsanado.

Por otra parte, nos ha llamado la atención el hecho de que los ítems enunciados en términos afirmativos (ítems 2,4,5,8 y 9l conllevan una asimetría clara respecto a los ítems que se realizan en términos negativos (ítems 1,3,6,7 y 10). En ellos se observa una tendencia a equilibrar los términos opuestos, lo cual a su vez puede deberse a que se dé una mayor confusión en la propia comprensión de la pregunta o bien por la propia situación de dicho ítem en la encuesta. Esto realmente es algo que quizás tendríamos que tener en cuenta en estudios posteriores en los que posiblemente seria oportuna la utilización de las pre- guntas en términos afirmativos, o bien, poner a prueba la hipótesis según la cual el nivel de formación académica puede establecer diferencias en los niveles de satisfacción simplemente por una cuestión de comprensión diferencial de la pregunta. En nuestro estudio, los usuarios del PLD, con mayor nivel cultural, muestran un mayor nivel de satisfacción.

Como ya ha sido referido, el TPQ es el primer cuestionario para la validación de la satisfacción respecto al proceso terapéutico en población en tratamiento por adicción a drogas desarrollado en Europa. Al menos dos estudios previos refieren criterios de fiabilidad interna y test-retest satisfactorios, si bien indican que las percepciones de tratamiento son fluctuantes en periodos breves de tiempo(7-8). Pese a ser uno de los cuestionarios que ha generado interés y publicaciones con posibilidades de generalización de uso, nos llama la atención que un cuestionario orientado hacia el modelo EFOM no incluya ítems relacionados con la accesibilidad, cuestiones ambientales, etc., y metodológicamente no permita segmentar a la población (situación judicial, tipo de programa en el que esta incluido,...) pues entendemos que las expectativas de un usuario en "cadena de cumplimiento" son distintas a la del que no lo está, las del que está en un centro ambulatorio a las del residencial, las del que tiene mayor arraigo social al que no lo tiene, las del heroinómano a las del cocainómano, etc.

Otro aspecto a destacar es la reticencia a contestar algunas de las variables planteadas, expresada por una alta ausencia de respuesta. En concreto, este efecto se hace notable en las variables referidas a la situación judicial y aquellas otras en las que se solicita al usuario haga un esfuerzo de memoria para emplazar cuestiones temporales, tales como cuándo se inició en el consumo de drogas, cuando inició el programa de tratamiento, etc. Sin embargo, este efecto de reticencia en la contestación no se ha producido en la ejecución delTPQ, lo cual nos hace suponer que resulta un cuestionario cómodo para el usuario al que no tiene reparos en contestar. Asimismo, el TPQ nos va a permitir, con un mínimo de requerimiento de personal y tiempo (3 minutos), obtener una visión más clara de cómo nos perciben los usuarios en los diferentes programas. Lo cual a corto y largo plazo puede beneficiar e indicar cuáles son los cambios que debemos ir introduciendo en el mismo. No hemos de olvidar que de cómo coincidan sus expectativas respecto al tratamiento puede moderarse el éxito del mismo, un individuo que no este satisfecho con el programa es más probable que abandone o de respuestas no esperadas.

Las encuestas de satisfacción comienzan a generalizarse durante los últimos años. Desde el punto de vista metodológico suelen ser incorrectas y sus resultados carecen de validez. A pesar de ello, es necesario elaborar un cuestionario o estándar de medición 
que nos permita obtener una visión más clara de cómo nos perciben los usuarios en los diferentes programas y compararnos inter e intra centros para ajustar los objetivos de gestión y funcionamiento del centro a las especificaciones de servicio de los usuarios, así como para valorar la pertinencia de los factores medidos. La disponibilidad de esta información junto con la de los indicadores de gestión y funcionamiento (numero y tipo de quejas, tiempos de tramitación, ...) nos permitirán prever las posibles tendencias y en función de ello implementar los mecanismos necesarios que permitan la corrección de un resultado no deseado. Es necesario seguir realizando investigaciones en esta línea, y el TPQ puede ser un instrumento útil para ello.

\section{BIBLIOGRAFÍA}

(1) Galgano A. Calidad Total. Madrid, Ediciones Díaz de Santos, 1993.

(2) ZeithamI V, Parasuraman A, Berry Y, Leonard L. Calidad Total en la Gestión de Servicios. Madrid, Ed. Díaz de Santos, 1993.
(3) James P. Gestión de Calidad Total. Madrid, Ed. Prentice Hall, 1997.

(4) Demming WE. Quality Productivity and Competitive Position. EUA, McGraw-Hill, 1992.

(5) Roure J, Rodríguez-Badal MA. Aprendiendo de los mejores: el modelo EFQM y el proceso de auto diagnóstico en la práctica. Gestión-2000 1999; 658.56 ROU.

(6) Pascoe CR. Patient satisfaction in primary healt care: A literature review and analysis. Eval Program Plann 1983; 6: 185-210.

(7) Marsden J, Stewart D, Gossop M, Rolfe A, Bacchus L, Griffiths P, Clarke K, Strang J. Assessing client satisfaction with treatment for substance use problems: Development of the Treatment Perceptions Questionnaire (TPQ). Addiction Research (in press).

(8) Mandersen J, Nizzoli U, Corbelli C, Margaron H, Torres MA, Castro del Prado I, Stewart D, Gossop M. Fiabilidad del "Maudsley addiction profile" (MAP, ERIT- Version) del "Treatment perceptions questionnaire" (TPQ) en Italia, España y Portugal para la evaluación de tratamientos. Adicciones 2001; 13: 217-227.

(9) Norusis MJ. Statistical Package for the Social Sciences. Release 9. SPSS Inc., Chicago, 2000. 